\title{
Categorías de riesgo según posturas adoptadas por los obreros de construcción civil de una empresa privada
}

\author{
Chávez-Torres Pamela ${ }^{1}$, Collantes-Villanueva Jeimy ${ }^{1}$, Maylle-Durand Katherine ${ }^{1}$
}

\section{RESUMEN}

Objetivo: determinar las categorías de riesgo según posturas adoptadas por los obreros de construcción civil. Material y métodos: estudio descriptivo de corte transversal. La población estuvo conformada por cien trabajadores de construcción civil de una empresa privada; la muestra estuvo conformada por 68 obreros que cumplieron con los criterios de selección. Luego se aplicó una historia ocupacional. Así mismo, se aplicó el método Ovako Working Analisys System (OWAS) para determinar la categoría de riesgo según las posturas adoptadas por los obreros de construcción civil durante la dinámica laboral. Resultados: las puntuaciones OWAS revelaron altos porcentajes de riesgo: 35,29\% presenta postura con efectos dañinos sobre el sistema músculo-esquelético con predominancia en la espalda $(57,35 \%)$ que obtuvo categoría de riesgo 2 (postura con posibilidad de causar daño al sistema músculoesquelético), y los brazos $(52,94 \%)$ con categoría de riesgo 2 (postura con posibilidad de causar daño al sistema músculo-esquelético). Discución: sobre las posturas adoptadas según las categorías de riesgo por sectores del cuerpo, el mayor riesgo de los obreros son los brazos y el tronco al realizar movimientos de rotación, flexión y extensión, principalmente en zona sacra, tronco y extremidades superiores. Un estudio similar, durante la evaluación de posturas, presentó zonas de riesgo como: tronco $63 \%$, brazos $60 \%$ y la zona de menor afectación y riesgo las piernas con $46 \%$. Conclusiones: según las posturas adoptadas por los obreros de construcción civil, el grupo predominante presenta la categoría de riesgo 3, es decir, posturas con efectos dañinos sobre el sistema músculo-esquelético.

Palabras clave: salud laboral, riesgos laborales, trabajadores. Perú. (Fuente DeCs BIREME).

\section{Risk categories positions taken by civilian construction workers of a private company}

\begin{abstract}
Objetive: To determine the risk category according to positions taken by civil construction workers. Material and Methods: Descriptive study of cross section. The population was conformed by 100 civil construction workers that were working in the company, the sample was conformed by 68 workers who met the selection criteria. Then applied an occupational history. The method Ovako Working Analysis System (OWAS) was also applied to determine category of risk according to the positions adopted by the workers of civil construction during labor dynamics. Results: OWAS scores revealed high percentages of risk: $35.29 \%$ present position with harmful effects on the muscular-skeletal system; being predominantly back $57.35 \%$ obtaining risk 2 category: position with the possibility of causing harm to the muscular-skeletal system and arms $52.94 \%$ category of risk 2: position with the possibility of causing damage to the muscularskeletal system. Discussion: On the positions adopted according to risk categories for areas of the body, the greatest risk of the workers are arms and trunk by rotation, flexion and extension movement mainly sacral area, trunk and upper extremities. A similar study for the evaluation of positions presented as risk areas: trunk $63 \%, 60 \%$ arms and the area of less involvement and risk legs 46\%. Conclusion: According to the positions taken by civil construction workers, the predominant group presents the category of risk 3: positions with harmful effects on the musculoskeletal system.
\end{abstract}

Key words: Occupational health, occupational hazards, workers. Peru. (Source DeCs BIREME).

1. Egresada de la Facultad de Enfermería. Universidad Peruana Cayetano Heredia. Lima - Perú. 


\section{INTRODUCCIÓN}

El Ministerio de Trabajo y Promoción Laboral define la ergonomía como: «La ciencia que busca optimizar la interacción entre el trabajador, máquina y ambiente de trabajo, con el fin de adecuar los puestos, ambientes y la organización del trabajo a las capacidades y limitaciones de los trabajadores, con el fin de minimizar el estrés y la fatiga, y con ello incrementar el rendimiento y la seguridad del trabajador» (1). Las posturas son el conjunto de actitudes posturales, dentro de la actividad laboral, encaminado a mantener una alineación de todo el cuerpo; el trabajador continuamente realiza esfuerzo físico durante la dinámica laboral, principalmente para mover el cuerpo, transportar objetos y mantener la postura; para responder a estas demandas el organismo realiza complejos mecanismos que finalizan en la contracción y relajación muscular, por ello, el objetivo de la higiene postural es aplicar medidas de protección, y reconocer consecuencias al adoptar una postura inadecuada previniendo así que aparezcan problemas músculo-esqueléticos (2).

Según el Ministerio de Salud, las condiciones laborales deben considerar factores individuales y el diseño de la zona de trabajo. La seguridad ocupacional comprende un conjunto de actividades para la protección del trabajador y la propiedad física de la empresa, todo ello mediante la prevención y el control de las acciones del hombre, de las máquinas y del medioambiente de trabajo, con la finalidad de prevenir lesiones (3). Para evaluar las posturas físicas adoptadas en el trabajo se debe tener en cuenta los factores relacionados con el entorno ocupacional: los trabajos que se hagan de forma repetitiva; los levantamientos de pesos; las posturas mantenidas en el trabajo, así como las rotaciones y flexiones, las cuales son perjudiciales para la salud. Las causas de adoptar posturas forzadas dependen, principalmente de factores relacionados con las condiciones de trabajo como el diseño de los puestos de trabajo; la organización del trabajo; la iluminación; las exigencias de las tareas tanto físicas como visuales; los conocimientos del trabajador, entre otros; es importante, como medida preventiva de lesiones músculo-esqueléticas, el mantener una correcta postura de trabajo (4).

Durante 32 años se ha utilizado el instrumento Ovako Working Posture Analysis System (OWAS) desarRollado por OVAKO Oy, en el Instituto de Salud Ocupacional en Finlandia, el cual es aplicado para determinar posturas y carga de trabajo. Se identifican cuatro categorías de riesgo: categoría de riesgo 1, postura normal; categoría de riesgo 2, postura con posibilidad de causar daños en el sistema músculo-esquelético; categoría de riesgo 3, pos- tura con efectos dañinos en el sistema músculo-esquelético, y categoría de riesgo 4, postura con efectos sumamente dañinos para el sistema músculo-esquelético (5).

La industria de la construcción es uno de los motores de la economía actual que encierra diversos riesgos ocupacionales en donde la salud de los trabajadores puede verse afectada muchos años después (6). El instituto Nacional de Estadística e Informática menciona en el Censo del 2007 que las personas que trabajan en el área de construcción civil representan el 18,8\% de la población económicamente activa (PEA) (7). En el marco normativo se menciona las recomendaciones internacionales de la Organización Internacional del Trabajo (OIT) para regular la seguridad, salud y bienestar en la industria de la construcción a nivel mundial, específicamente, en las obras de construcción y edificación deberían planearse, prepararse y aplicarse estrictamente las normas para prevenir los riesgos que puedan presentarse en el ambiente laboral, evitar en el trabajo posturas y movimientos excesiva o innecesariamente fatigosos, organizar el trabajo teniendo en cuenta la seguridad y la salud de los trabajadores, utilizar materiales o productos apropiados desde el punto de vista de la seguridad y de la salud, y llevar un control de los indicadores de salud ocupacional (8). Wolfgang y Vedder mencionan que no se dispone de una relación completa de la normativa existente sobre la evaluación de las posturas, pero que en el avance de la salud ocupacional existen las guías del NIOSH sobre levantamiento de pesos (NIOSH 1981) se refieren a los límites de peso para carga que contribuyen en la mejora de la calidad laboral (9).

La consecuencia de las posturas de trabajo inadecuadas son los problemas músculo-esqueléticos, de aparición lenta y carácter inofensivo en apariencia, por lo que se ignoran hasta que el daño se presenta en forma crónica (10). Las posturas estáticas de trabajo son posiciones isométricas, en las que ocurre poco movimiento, y que causan sobrecarga muscular; se producen en todos los trabajos que requieren pasar tiempos prolongados de pie o sentado, y tienen influencia probada en el desarrollo de problemas lumbares y cérvico-braquiales, como es el caso de los obreros de construcción civil quienes presentan largas jornadas laborales en posiciones de riesgo para la sa$\operatorname{lud}(11,12)$.

El objetivo fundamental de la aplicación de esta herramienta en la enfermería ocupacional es constituir una estructura que pueda cubrir las necesidades del trabajador partiendo de la valoración que se conceptualiza en la exploración e identificación de categorías de riesgo postural 
durante la dinámica laboral. La enfermería, mediante un enfoque basado en medidas preventivas en la salud pública y ocupacional, realiza una importante labor para garantizar el bienestar del obrero, siendo la evaluación de las posturas la especialidad que tienen como propósito adecuar las actitudes de la persona frente a la ocupación $(13,14)$. El presente artículo tiene como objetivo determinar la categoría de riesgo según posturas adoptadas por los obreros de construcción civil.

\section{MATERIAL Y MÉTODOS}

Estudio descriptivo de corte transversal, a través del cual se determinaron las categorías de riesgo según posturas adoptadas por los obreros de construcción civil. La investigación se llevó a cabo en una empresa privada de construcción civil situada en el distrito de San Borja, LimaPerú. La población de estudio estuvo conformada por cien trabajadores de construcción civil que laboraban en la empresa. Se utilizó la fórmula de cálculo muestral con población finita para determinar el número de participantes en el estudio, el resultado fue de 36 obreros.

Para delimitar la muestra se revisó la historia clínica proporcionada por el ingeniero de seguridad de 80 obreros de construcción, para verificar que no tuvieran problemas músculo-esqueléticos; que se encuentren en el intervalo de edad entre 20 y 50 años, y presenten un mínimo de 2 años de antigüedad laborando en la ocupación, según la planilla de pagos. Se realizó la firma del consentimiento informado para verificar quiénes tenían voluntad de participar del estudio. Previamente se explicó todos los aspectos éticos que intervienen en la investigación. Finalmente, la muestra estuvo integrada por 68 obreros de construcción civil que cumplían con los criterios mencionados.

\section{Técnicas e instrumentos de la recolección de datos}

Se aplicó como instrumento una historia ocupacional para tener datos característicos de la población en estudio, que fue adaptado por los investigadores del documento original elaborado por Farro Peña et al. (15). Posteriormente se aplicó el método OWAS, como instrumento para determinar la categoría de riesgo según posturas adoptadas por los obreros de construcción civil, el instrumento en mención ha sido validado a nivel internacional por la Organización Internacional del Trabajo (OIT) con una confiabilidad de 0,93 (16). El instrumento basa sus resultados en la observación de las diferentes posturas adoptadas por el trabajador durante el desarrollo de la tarea, de las combinaciones de espalda, brazos, piernas y la manipulación de carga. El método propone la codificación de las posturas según los puntajes identificados en la espal- da, brazo, piernas y la carga manipulada; los cuales se codifican según la propuesta del instrumento OWAS para identificar la categoría de riesgo en general.

Finalizada la fase de codificación de las posturas y conocidas las categorías de riesgo propuestas por el método OWAS, se procedió a la asignación de la categoría de riesgo correspondiente a cada código de postura. La categoría de riesgo para cada posible combinación de la posición de espalda, brazos y piernas y de la carga manipulada. Siendo clasificadas en cuatro categorías de riesgo en base al efecto potencial sobre el sistema músculo esquelético:

$>$ Categoría de riesgo 1: postura sin efectos dañinos sobre el sistema músculo-esquelético.

$>$ Categoría de riesgo 2: postura con posibilidad de causar daño sobre el sistema músculo-esquelético.

$>$ Categoría de riesgo 3: postura con efectos dañinos sobre el sistema músculo-esquelético.

$>$ Categoría de riesgo 4: postura con efectos sumamente dañino sobre el sistema músculo-esquelético.

Una vez calculada la categoría de riesgo en general, el método OWAS propone la clasificación del efecto potencial de las posturas sobre el sistema músculo-esquelético según sectores del cuerpo humano, lo que contempla el análisis de las frecuencias relativas de las diferentes posturas calculando el número de veces que se repite cada posición en relación al tiempo de observación. Luego de realizar el cálculo se determinó la categoría de riesgo por sectores del cuerpo utilizando la gráfica de «Clasificación de las categorías de riesgo de las posiciones del cuerpo según su frecuencia relativa». Los valores del riesgo calculados para cada posición permitieron identificar aquellos sectores del cuerpo humano que presentan un mayor efecto potencial sobre el sistema músculo-esquelético.

Para el presente estudio se solicitó los permisos correspondientes al gerente general de la empresa y se coordinó con los ingenieros de seguridad y de campo para la asignación de facilidades requeridas durante la aplicación de los instrumentos. Se recolectaron los datos durante una semana en los horarios de martes a viernes de 8:00. a 18:00 h. y sábado de 8:00 a 13:00 h. en una oficina asignada para la aplicación de la historia ocupacional. La obra estaba dividida en cinco sectores los cuales estratégicamente sirvieron para organizar los tiempos durante la ejecución por los investigadores, se evaluó un sector por día, dichos sectores son: carpintería, banco, encofrado, sector eléctrico y ferrería. A cada obrero se le preguntó su nombre para verificar en su historia clínica el cumplimiento de los criterios de selección mencionados anteriormente. Cabe 
mencionar que dichas historias clínicas fueron proporcionadas por la empresa y tenían una antigüedad no mayor a un 1 mes. De 80 evaluados se seleccionó la muestra conformada por 68 obreros.

Posteriormente, se aplicó el consentimiento informado donde los participantes fueron informados de todos aquellas consideraciones éticas que se involucran en el estudio, el obrero de construcción, aprobaba su participación con la firma del documento, y seguidamente se le aplicaba la historia ocupacional. Luego, los investigadores procedieron a la aplicación del método OWAS, el cual consistió en la observación de la dinámica laboral en el turno diurno; se observó a cada trabajador a una distancia entre 10 y 20 metros durante 20 minutos sin que este tenga conocimiento de que se le estuvo evaluando para así evitar que el obrero modifique su postura rutinaria pudiendo generar datos incorrectos. A las posturas identificadas en cada trabajador se le asignó un puntaje correspondiente; de acuerdo al método OWAS los puntajes son:

Postura de espalda

$>1$ Espalda derecha.

$>2$ Espalda doblada.

$>3$ Espalda con giro.

$>4$ Espalda doblada con giro.

Postura de brazos

$>1$ Los dos brazos bajos.

$>2$ Un brazo bajo y el otro elevado.

$>3$ Los dos brazos elevados.

Postura de piernas

$>1$ Sentado.

$>2$ De pie con las dos piernas rectas con el peso equilibrado entre ambas.

$>3$ De pie con una pierna recta y la otra flexionada con el peso desequilibrado entre ambas.

$>4$ De pie o en cuclillas con las dos piernas flexionadas y el peso equilibrado entre ambas.

$>5$ De pie o en cuclillas con las dos piernas flexionadas y el peso desequilibrado entre ambas.

$>6$ Arrodillado.

$>7$ Andando.

Así mismo, se asignó el puntaje de la carga manipulada, siendo los puntajes

$>1$ Carga menor a $10 \mathrm{~kg}$.

$>2$ Carga entre 10 y $20 \mathrm{~kg}$.

$>3$ Carga de peso mayor a $20 \mathrm{~kg}$.

A continuación se utilizó el gráfico «Clasificación de las categorías de riesgo de los códigos de postura» aplicando los puntajes asignados a la postura de espalda, brazos, piernas y manipulación de cargas para identificar la categoría de riesgo en general de cada obrero. Posteriormente, de la postura observada se estimó la frecuencia relativa que se obtuvo de la razón del número de repeticiones de la postura observada sobre el número de posturas totales, se puede observar el registro de las frecuencias de cada obrero de construcción civil.

Para determinar las categorías de riesgo por sectores del cuerpo humano; utilizando la estimación de la frecuencia relativa en combinación con los puntajes obtenidos de brazos piernas y espalda, se identificó la categoría de riesgo según posturas de espalda brazos y piernas empleando el gráfico «Clasificación de las categorías de riesgo de las posiciones del cuerpo según su frecuencia relativa». Finalmente, en el programa Microsoft Excel versión 2007 se elaboró una hoja de cálculos con la base de datos obtenidos a fin de elaborar tablas y gráficos de frecuencia.

\section{RESULTADOS}

En la tabla 1, el 52,94\% utiliza una posición de espalda doblada durante la dinámica laboral; un $8,82 \%$ utiliza la posición de espalda doblada con giro; así mismo, en la valoración de la postura de los brazos el $50 \%$ realiza las actividades con un brazo elevado y el otro por debajo del hombro, también se observa que el $27,94 \%$ labora con una postura de piernas andando.

En la tabla 2, el 80,88\% (55 obreros) del total, manipulan cargas por debajo de $10 \mathrm{~kg}$ de peso y un $5,88 \%$ (cuatro obreros) supera los $20 \mathrm{~kg}$ de carga.

En la tabla 3, con relación a la categoría de riesgo localizados en zonas del cuerpo, el 57,35\% (39 obreros) presenta una categoría de riesgo 2: con una postura de espalda con posibilidad de causar daño al sistema músculoesquelético; en los brazos se observa que el 52,94\% (36 obreros) también presenta una categoría de riesgo 2: con una postura con posibilidad de causar daño al sistema músculo-esquelético; con relación a las piernas el 41,18\% (28 obreros) se caracteriza por presentar una categoría de riesgo 1: con una postura sin efectos dañinos en el sistema músculo-esquelético.

En la tabla 4, el 35,29\% (24 obreros) utiliza posturas con efectos dañinos sobre el sistema músculo-esquelético; el $5,88 \%$ (cuatro obreros) presenta la máxima categoría de riesgo donde la carga causada por la postura tiene efectos sumamente dañinos sobre el sistema músculo-esquelético. 
Tabla 1. Posturas de espalda, brazos y piernas adoptadas por los obreros de construcción civil según método OWAS Lima, junio de 2013

\begin{tabular}{|c|c|c|c|}
\hline & Posturas & $\mathbf{N}$ & $\%$ \\
\hline \multirow{4}{*}{ 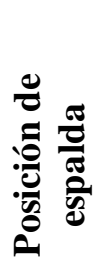 } & Espalda doblada & 36 & 52,94 \\
\hline & Espalda con giro & 15 & 22,06 \\
\hline & Espalda derecha & 11 & 16,18 \\
\hline & Espalda doblada con giro & 6 & 8,82 \\
\hline \multirow{5}{*}{ 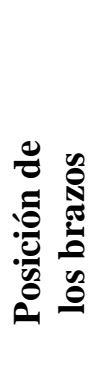 } & Total & 68 & 100,00 \\
\hline & Los brazos bajos & 31 & 45,59 \\
\hline & Un brazo bajo y el otro elevado & 34 & 50,00 \\
\hline & Los dos brazos elevados & 3 & 4,41 \\
\hline & Total & 68 & 100,00 \\
\hline \multirow{8}{*}{ 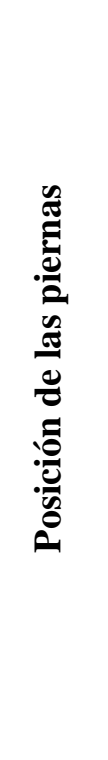 } & Andando & 19 & 27,94 \\
\hline & $\begin{array}{l}\text { De pie con una pierna recta y la otra flexionada } \\
\text { con el peso desequilibrado entre ambas }\end{array}$ & 17 & 25,00 \\
\hline & $\begin{array}{l}\text { De pie o en cuclillas con las dos piernas } \\
\text { flexionadas y el peso equilibrado entre ambas }\end{array}$ & 10 & 14,71 \\
\hline & $\begin{array}{l}\text { De pie o en cuclillas con las dos piernas } \\
\text { flexionadas y el peso desequilibrado entre } \\
\text { ambas }\end{array}$ & 9 & 13,24 \\
\hline & Sentado & 6 & 8,82 \\
\hline & $\begin{array}{l}\text { De pie con las piernas rectas con el peso } \\
\text { equilibrado entre ambas }\end{array}$ & 4 & 5,88 \\
\hline & Arrodillado & 3 & 4,41 \\
\hline & Total & 68 & 100,00 \\
\hline
\end{tabular}

Tabla 2. Carga manipulada durante la dinámica laboral por los obreros de construcción civil, Lima, junio 2013

\begin{tabular}{lcc}
\hline Cargas Soportadas & N & $\%$ \\
\hline & 55 & \\
Menos de $10 \mathrm{~kg}$ & 9 & 80,88 \\
Entre 10 y $20 \mathrm{~kg}$ & 4 & 13,24 \\
Más de $20 \mathrm{~kg}$ & 68 & 5,88 \\
Total & 68 & 100,00 \\
\hline
\end{tabular}


Tabla 3. Categoría de riesgo según sectores del cuerpo en espalda, brazos y piernas de los obreros de construcción civil. Lima, junio 2013

\section{Categoría de riesgo en espalda}

\begin{tabular}{|c|c|c|c|}
\hline C.R. & & $\mathrm{N}$ & $\%$ \\
\hline 1 & Postura sin efectos dañinos en el sistema músculo-esquelético & 11 & 16,18 \\
\hline 2 & $\begin{array}{l}\text { Postura con posibilidad de causar daño al sistema músculo- } \\
\text { esquelético }\end{array}$ & 39 & 57,35 \\
\hline 3 & Postura con efectos dañinos sobre el sistema músculo -esquelético & 17 & 25,00 \\
\hline 4 & $\begin{array}{l}\text { La carga causada por la postura tiene efectos sumamente dañinos } \\
\text { sobre el sistema músculo-esquelético }\end{array}$ & 1 & 1,47 \\
\hline \multicolumn{4}{|c|}{ Categoría de riesgo en brazos } \\
\hline C.R. & & $\mathrm{N}$ & $\%$ \\
\hline 1 & Postura sin efectos dañinos en el sistema músculo-esquelético & 31 & 45,59 \\
\hline 2 & $\begin{array}{l}\text { Postura con posibilidad de causar daño al sistema músculo- } \\
\text { esquelético }\end{array}$ & 36 & 52,94 \\
\hline 3 & Postura con efectos dañinos sobre el sistema músculo -esquelético & 1 & 1,47 \\
\hline 4 & $\begin{array}{l}\text { La carga causada por la postura tiene efectos sumamente dañinos } \\
\text { sobre el sistema músculo-esquelético }\end{array}$ & 00 & 0,00 \\
\hline \multicolumn{4}{|c|}{ Categoría de riesgo en piernas } \\
\hline C.R. & & $\mathrm{N}$ & $\%$ \\
\hline 1 & Postura sin efectos dañinos en el sistema músculo-esquelético & 28 & 41,18 \\
\hline 2 & $\begin{array}{l}\text { Postura con posibilidad de causar daño al sistema músculo- } \\
\text { esquelético }\end{array}$ & 20 & 29.41 \\
\hline 3 & Postura con efectos dañinos sobre el sistema músculo -esquelético & 19 & 27,94 \\
\hline \multirow[t]{2}{*}{4} & $\begin{array}{l}\text { La carga causada por la postura tiene efectos sumamente dañinos } \\
\text { sobre el sistema músculo-esquelético }\end{array}$ & 1 & 1,47 \\
\hline & Total & 68 & 100,00 \\
\hline
\end{tabular}

Tabla 4. Categoría de riesgo general de los obreros de construcción civil. Lima, junio 2013

\begin{tabular}{|c|c|c|c|}
\hline \multicolumn{4}{|c|}{ Categoría de riesgo general } \\
\hline C.R. & & $\mathrm{N}$ & $\%$ \\
\hline 1 & Postura sin efectos dañinos en el sistema músculo-esquelético & 20 & 29,41 \\
\hline 2 & $\begin{array}{l}\text { Postura con posibilidad de causar daño al sistema músculo- } \\
\text { esquelético }\end{array}$ & 20 & 29,41 \\
\hline 3 & $\begin{array}{l}\text { Postura con efectos dañinos sobre el sistema músculo - } \\
\text { esquelético }\end{array}$ & 24 & 35,29 \\
\hline \multirow[t]{2}{*}{4} & $\begin{array}{l}\text { La carga causada por la postura tiene efectos sumamente } \\
\text { dañinos sobre el sistema músculo-esquelético }\end{array}$ & 4 & 5,88 \\
\hline & Total & 68 & 100,00 \\
\hline
\end{tabular}




\section{DISCUSIÓN}

En el estudio realizado en una obra de construcción civil en el distrito de San Borja existe una población de 100 obreros que se dedican a la edificación. La presente investigación se concentra en la actividad laboral de una muestra representativa conformada por 68 obreros que cumplieron con los criterios de selección antes mencionados, quienes en el día a día presentan exposición a riesgos posturales propios de la dinámica laboral. En la Tabla 1 se observó que el 52,94\% utiliza una posición de espalda doblada durante la dinámica laboral; un $8,82 \%$ utiliza la posición de espalda doblada con giro; así mismo, en la valoración de la postura de los brazos, el 50\% realiza las actividades con un brazo elevado y el otro por debajo del hombro. También se ve que el $27,94 \%$ labora con una postura de piernas andando. Según Bellorín, quien en el año 2007 evalúa las lesiones músculo-esqueléticas según regiones anatómicas, menciona que las más afectadas son las relacionadas con la postura: espalda inferior $(50,60 \%)$; hombros $(13,25 \%)$; cuello y espalda superior $(8,43 \%$ cada una), y rodillas $(6,02 \%)$; los síntomas de espalda inferior fueron referidos principalmente por los obreros (17).

Los obreros de construcción civil están inmersos en una dinámica laboral que implica realizar actividades físicas caracterizadas por jornadas diarias extremadamente duras, agregado a las exigencias de la empresa para aumentar la producción en el mínimo tiempo posible, eso les conlleva adoptar posturas perjudiciales, se evidenció a parte del personal utilizando un arnés de protección, colgados para armar una columna, otros con flexión de toda la espalda, movimientos rotativos del tronco, entre los más resaltantes que repercuten en problemas músculo-esqueléticos.

En cuanto a la manipulación manual de la carga, se observa que el $80,88 \%$ realiza un esfuerzo de carga por debajo de los $10 \mathrm{~kg}$ de peso y 5,88\% superan los $20 \mathrm{~kg}$ de carga en la tabla 2. Diferente a los resultados que encontró Schulz donde se observa que $46 \%$ carga diariamente pesos que oscilan entre 10 y $19 \mathrm{~kg}$ (18).

La edificación, dependiendo de las estructuras, presenta una manipulación de carga diferente, se evidenció que la presente obra cumplía con los estándares de la Organización Internacional del trabajo (OIT) y con la normativa ISO 9000, que propone la gestión de la calidad para garantizar las condiciones del trabajo, el cual determina la medición de riesgos laborales y el control del personal de la empresa (19); es por ello que se observa un menor riesgo en la manipulación de la carga, no obstante, es de sumo cuidado debido a que de presentarse de forma repetitiva puede aumentar la probabilidad de presentar una lesión músculo-esquelética. En relación a la categoría de riesgo localizados en zonas del cuerpo, en la Tabla 3 se identifica un 57,35\% que presenta una categoría de riesgo 2: con una postura de espalda con posibilidad de causar daño al sistema músculo-esquelético; en cuanto a los brazos se observa que el 52,94\% también presenta una categoría de riesgo 2: con una postura con posibilidad de causar daño al sistema músculo-esquelético; con relación a las piernas, el $41,18 \%$ se caracteriza por presentar una categoría de riesgo 1: con una postura sin efectos dañinos en el sistema músculo-esquelético. Similar a lo descrito por Montiel etal. quienes en la valoración de la carga postural mencionan que la zona de mayor riesgo es tronco en $63 \%$, los brazos $60 \%$, y que la zona de menor afectación y riesgo son las piernas con un puntaje de $46 \%$ (20).

Así mismo, Varela en el diagnóstico de las estaciones de trabajo de una empresa identifica que el $100 \%$ de los obreros presenta una categoría de riesgo 4 en el sector de espalda, el $100 \%$ presenta una categoría de riesgo 3 en el sector de los brazos, y el $100 \%$ presenta una categoría de riesgo 2 en las piernas (21). Según las categorías de riesgo por sectores en el cuerpo, el mayor uso por parte de los obreros son lo brazos y el tronco realizando movimiento de rotación, flexión y extención principalmente la zona sacra, tronco y extremidades superiores; se identifica mayor riesgo en los sectores mencionados. Se puede determinar que ambos estudios presentan resultados semejantes motivo por el cual existe un aumento del riesgo de lesiones músculo-esqueléticas en los obreros de construcción civil.

En la tabla 4 se observa que el $35,29 \%$ de los obreros presenta una categoría de riesgo 3 , donde utilizan posturas con efectos dañinos sobre el sistema músculo-esquelético. Tal como lo menciona Vargas en su estudio, donde coincide con las posturas mencionadas anteriormente y concluye que la categoría de riesgo predominante es la tercera categoría, quienes utilizan posturas con efectos dañinos sobre el sistema músculo-esquelético (22). Como se evidencia en las posturas, y la probabilidad de daño para la salud que representa durante la dinámica de los obreros de construcción civil, las actividades y exigencias inmersas se consideran potencialmente en las lesiones músculo-esqueléticas a corto, mediano y largo plazo, lo que indica que el área de construcción civil, por ser una ocupación con alta peligrosidad por los riesgos que comprende, la probabilidad de presentar lesiones es de consideración y a tomar en cuenta en investigaciones futuras. 


\section{REFERENCIAS BIBLIOGRÁFICAS}

1. Villasante AJ. Sistema Informativo de Información Laboral. Mintra [Internet]. 2008 Nov [citado 22 jun 2013]; 375 (1): [aprox. 19 pag.]. Disponible en: http:/ /www.mintra.gob.pe/archivos/file/SNIL/normas/ 2008-11-28_375-2008-TR_1399.pdf

2. 2. Villar FM. Posturas de trabajo: evaluación del riesgo. INSHT [Internet]. 2011 [citado 22 jun 2013]; 1 (1): [aprox. 6 pag.]. Disponible en: http:// www.insht.es/MusculoEsqueleticos/Contenidos/ Formacion\% 20divulgacion/material\%20didactico/ Posturas\% 20trabajo.pdf

3. Ministerio de Salud. Manual de Salud Ocupacional. MINSA [Internet]. 2005 [citado 22 jun 2013]. [Aprox. 97 pag.]. Disponible en: http://www.digesa.minsa. gob.pe/publicaciones/descargas/manual_deso.PDF

4. Ministerio del trabajo. Seguridad durante la construcción. Mintra [Internet]. 2007 [citado el 26 de Jul 2011]; 2 (4): [Aprox. 11 pag.]. Disponible en: http:/ /www.ila.org.pe/actividades/docs/seminario_mintra/ exp_seg_ construccion_2007.pdf

5. Montes de Oca MM., Diagnóstico ergonómico de los trabajadores en la industria de la construcción [tesis]. México D.F. Instituto Politécnico Nacional; 2007.

6. Bernard BC. A critical review of epidemiologic evidence for work-related musculoskeletal disorders of the neck, upper extremity and low back. NIOSH [Internet]. 1997. [citado el 03 de junio 2011];97(141): [aprox. 23 pág.] .Disponible en: http://www.cdc.gov/ niosh/docs/97- 141/

7. INEI.gob[Internet].Lima: Instituto Nacional de Estadìstica e Informàtica (INEI); c2011-01 [citado el 1 de julio 2011- actualizado 13 de agosto de 2013]. Disponible en: www.inei.gob.pe

8. Manual para la identificación y evaluación de riesgos laborales. [Internet]. Dic 2006 [citado el 1 de julio 2011];10 (1):[aprox. 187 pag.]. Disponible en : http:// www.academia.edu/1216408/DIAGNOSTICO_ER GONOMICO_DE_LOS_TRABAJADORES_EN _LA_INDUSTRIA_DE_LA_CONSTRUCCION

9. Wolfgang L, Vedder J, Ergonomía. Enciclopedia de salud y seguridad en el trabajo. [Internet]. [citado el 1 de julio 2011] Disponible en: http://www.insht.es/ InshtWeb/Contenidos/Documentacion/TextosOnline/ EnciclopediaOIT/ tomo1/29.pdf

10. Moreno JB, Peñacoba PC, Araujo GV. Ergonomía y Psicología. Programa técnico en prevención de riesgos laborales. OIT [Internet]. 2008 [citado el 26 de julio 2013]. Disponible en: http://white.oit.org.pe/spanish/ 260ameri/ oitreg/activid/proyectos/actrav/edob/ expeduca/pdf/0630331.pdf
11. Sanz L, Gonzales J, Navarrete F, Martínez E. Estudio ergonómico del cirujano durante la colecistectomía por vía abierta y laparoscópica. Cir Esp [Internet]. 2002 [citado 18 de abril de 2013]; 71(4): [aprox. 110 pág.]. Disponible en: http://www.elsevier.es/en/node/ 2017054

12. Ministerio del Trabajo. Regimen laboral en construcción civil. 2013. Mintra [Internet]. [citado el 26 de julio 2013] www.mintra.gob.pe/contenidos/drt/ servicios/REGIMEN_LABORAL.ppt

13. Parra M. Conceptos básicos en la Salud Laboral. CUTC [Internet]. May 2003 [citado el 16 de septiembre 2012]; 1(9): [aprox. 31 pág.]. Disponible en: http://www.oitchile.cl/pdf/publicaciones/ser/ ser009.pdf

14. Instituto Nacional de Seguridad e Higiene en el trabajo. Manipulación de Cargas. INSHT [Internet]. 2003 [citado el 26 de julio 2013] 1; (97): [Aprox. 60 pág.]. Disponible en: http://www.insht.es/InshtWeb/ Contenidos/ Normativa/GuiasTecnicas/Ficheros/ cargas.pdf

15. Farro PG., Bolaños GH., Musayón OY., Pecho MI. Chávez A.A., Campos C.H., Dávila C., Evaluación del plan de cuidados sanitarios sobre salud ocupacional de familias que trabajan en el reciclado de basura en Lomas de Carabayllo. Rev Enferm Herediana 2008; 1 (2): 119-122.

16. Karhu O. Observing working postures in industry: Exampes of OWAS application. Appl Ergon. 1981; 12 (1): 7-13.

17. Bellorín M, Sirit Y, Rincón C. Amortegui M., Síntomas Músculo Esqueléticos en trabajadores de una empresa de construcción Civil. Salud de los Trabajadores [Internet]. Dic. 2007 [citado el 16 de septiembre 2012]; 15 (2): [Aprox. 10 pag.].Disponible en: http:// www. scielo.org.ve/scielo.php? pid $=$ S 1315 01382007000200003\&script=sci_arttext

18. Schulz GK. Programa de higiene postural para la prevención de las lesiones de la columna vertebral. Caso: trabajadores del sector eléctrico. [Internet]. Dic 2008. [citado el 16 de septiembre 2012]; 4 (7): [Aprox. 46 pag.]. Disponible en: http:// www.cidar.uneg.edu.ve/DB/bcuneg/EDOCS/TESIS/ TESIS_POSTGRADO/ESPECIALIZACIONES/ S A L U D _ O C U P A C I O N A L / T G E R R 64D452010RojasDenis.pdf

19. Vargas ZF. La gestión de la calidad en la formación profesional. OIT [Internet]. 2003. [citado el 28 de junio de 2013]; 1 (12): [aprox. 46 pág.]. Disponible en: http://www.sld.cu/galerias/pdf/sitios/infodir/ calidad.pdf 
20. Monitel M., Romero J., Lubo PA., Uqvedo AL., Rojas L., Betulio C.H., Sanabria C.H., Valoración de la carga postural y riesgo musculo esquelético en trabajadores de una empresa metalmecánica. Salud de los trabajadores [Internet]. 2006 [citado el 28 de junio de 2013]; 14(1): [Aprox. 8 pág.]. Disponible en: http:/ /dialnet.unirioja.es/ servlet/articulo?codigo $=2046054$

21. Varela CR., Diagnóstico de la estación de trabajo, de recepción e inspección de cartera de huevo del área de producción en una empresa moldeadora de celulosa. Ergonomía ocupacional [Internet]. Dic 2007 [citado el 28 de junio de 2013]; 1 (30): [Aprox. 12 pág.]. Disponible en: http://www.ergocupacional.com/ 4910/58967.html
22. Franco V.J., Evaluación de riesgos ergonómicos y psicosociales para la construcción de aceras en promoción de viviendas [tesis]. Esp. Universidad de La Rioja; 2012.

\section{Correspondencia \\ Jeimy Collantes Villanueva \\ Miguel Baquero 251, Lima. \\ Telefóno: 4314731 - 4244731 \\ Correo electrónico: jeimy.collantes@upch.pe}

Forma de citar este artículo: Chávez-Torres P, CollantesVillanueva J, Maylle-Durand K. Categorías de riesgo según posturas adoptadas por los obreros de construcción civil de una empresa privada. Rev enferm Herediana. 2013;6(1):25-33. 